\title{
Enhanced mechanical energy extraction from transverse galloping using a dual mass system
}

\author{
D. Vicente-Ludlam, A. Barrero-Gil *, A. Velazquez \\ Aerospace Propulsion and Fluid Mechanics Department, School of Aeronautics, Universidad Politecnica de Madrid, Plaza Cardenal Cisneros \\ 3, E-28040 Madrid, Spain
}

\begin{abstract}
A B S T R A C T
This paper offers a theoretical study of energy extraction through transverse galloping using a dual-mass system. To this end, a two-degree-of-freedom model is developed where fluid forces on the galloping body are described resorting to quasi-steady hypothesis; the model is solved approximately by using the Harmonic Balance Method. Three possible configurations of the dual-mass system have been analyzed. Two of them show an improvement in the efficiency of energy extraction with respect to that of the single mass configuration when the mechanical properties of the dual-mass system are appropriately chosen. In addition, the dual-mass system promotes a broadening of the values of the incident flow velocities at which the efficiency is kept high.
\end{abstract}

\section{Introduction}

The interaction of an elastic bluff body with a fluid flow can lead to significant flow-induced vibrations, like vortexinduced vibrations, transverse galloping, flutter or buffeting. These flow-induced vibrations are usually considered to be an undesirable effect for civil or marine structures as they can reduce their lifetime by fatigue loading, compromise their service, or put at risk their integrity. However, it is also possible to take advantage of them by extracting useful energy from the fluid flow. The basic idea is to convert part of the flow's kinetic energy into oscillatory mechanical energy of the body. Afterwards, the mechanical energy of the body may be converted into electrical energy by electromagnetic, piezoelectric, or electrostatic means.

Several concepts introduced in the last decade work in this direction and should be referenced here. From a fundamental point of view, where the flow-induced vibration phenomenon is analyzed without any specific transduction system, we must mention the work of Bernitsas et al. [1] who described a way to use the oscillations induced by vortex shedding from a spring-mounted circular cylinder under the action of a water current. The influence of some key parameters like the mass ratio, the mechanical damping, the Reynolds number [2], and the cylinder's aspect ratio (length to diameter ratio) in the electrical power was experimentally investigated in a re-circulating water channel. VIV potential for energy extraction has also been studied by Grouthier et al. [3], Mackowski and Williamson [4], Barrero-Gil et al. [5], or Sanchez-Sanz et al. [6]. In the last two years, several research has been carried out considering a piezoelectric material as a transduction means to produce small quantities of electrical power (of the order of milli-watts) from VIV of a circular cylinder under the action of an airstream. For example, Abdelkefi et al. [7] presented a theoretical analysis using a wake-oscillator model to describe

\footnotetext{
* Corresponding author.

E-mail address: antonio.barrero@upm.es (A. Barrero-Gil).
} 
fluid forces on the oscillating circular cylinder which is appropriately coupled with the cylinder and piezoelectric dynamics. Additional theoretical and numerical research can be found in Mehmood et al. [8] and Dai et al. [9,10], as well as in Akaydin et al. [11] where an experimental approach is also given.

Energy harvesting from oscillations of a circular cylinder in the wake of a fixed upstream cylinder has been experimentally studied by Nishi et al. [12] and by Jung and Lee [13], where electromagnetic conversion was used. Abdelkefi et al. [14] carried out experiments to investigate the effects of wake galloping on the range of flow speeds over which a galloping-based piezo-aeroelastic energy harvester can be effectively used. Two different upstream cylinders and a wide range of spacing between the upstream and downstream cylinders were considered.

The use of flutter phenomena for energy extraction has also been studied by Tang et al. [15] or Zhu et al. [16]. Modeling and experimental results are given by Bryant and Garcia [17], where piezoelectric conversion was employed. Theoretical extensions can be found in Abdelkefi et al. [18] or Bae et al. [19], where structural and aerodynamic nonlinearities are considered in the piezoaeroelastic system, and in Abdelkefi et al. [20] where a cambered wing-based is considered. Finally, the energy-harvesting eel proposed by Allen and Smits should be mentioned, where a piezoelectric membrane is placed in the wake of a bluff body [21,22]. When the mass and elastic properties of the membrane are appropriately chosen, the largescale vortex street formed behind the body induces significant oscillations in the membrane that can be converted into electricity.

The idea of using transverse galloping (TG) as a way of extracting energy from a flow was investigated in Barrero-Gil et al. [23]. They employed a quasi-steady approximation to model TG fluid forces and found an analytical expression for the energy transferred from the flow to the galloping body as a function of the mass ratio, the mechanical damping, the reduced velocity, and the geometry of the body. TG potential for energy harvesting was further investigated theoretically, numerically, and experimentally by Abdelkafi and co-workers [24-26], Sirohi and Mahadik [27], or Zhao et al. [28]. Briefly speaking, TG is a motion-induced instability that appears in some elastic bluff bodies when the velocity of the incident flow exceeds a critical value. Then, the stabilizing effect of mechanical damping is overcome by the destabilizing effect of the fluid force. A small transverse displacement of the body induces an angle of attack relative to the incoming flow and a fluid force appears in the direction of the displacement in such a way that energy is pumped from the current to the body. Oscillatory motion (mainly transverse to the flow) develops with increasing amplitude until the energy dissipated per cycle by mechanical damping balances the energy input per cycle from the flow. If the geometry of the body and the elastic properties are appropriate, instability may appear at low flow velocity and with high excitation amplitude, making TG a very promising way for harvesting energy successfully. For a comprehensive introduction to TG the reader is referred to Parkinson [29], Blevins [30], Naudascher and Rockwell [31], or Paidoussis et al. [32].

The use of a dual mass system to enhance the energy extraction from vibration sources was first proposed by Tang et al. [33]. They considered the case of a linear vibrating system where one of the masses was excited by an external oscillating force. Using the concept of a dual mass system, Nishi [34] proposed to enhance the power extraction from vortex-induced vibrations carrying out a theoretical study to examine the energy extraction through VIV from a dual mass system. VIV fluid forces on the main mass were described by a wake oscillator model finding enhancement of power extraction by the dual mass system for appropriate combination of the dual mass parameters.

Here the possibility of enhancing the energy extraction is discussed as well as its efficiency using TG by means of a dual mass system. As it will be shown in detail later, in the dual mass system a secondary mass (not exposed to the flow) is elastically connected to the galloping body whose oscillation causes the secondary mass to oscillate. By correctly matching the secondary mass and the stiffness of the elastic connection between both masses, additional energy can be harvested from the flow; thereby increasing the overall system efficiency. Three possible dual-mass configurations will be studied hereafter. They are characterized by the location of the generator used to extract energy. In the first configuration ( $\mathrm{C} 1$ ), the generator was placed between the secondary mass and a fixed wall; in the second configuration (C2), the generator was located in between the main and the secondary masses. The third configuration (C3) situated the generator in between a fixed wall and the primary mass (see Fig. 1).

The main goal of this study is to investigate the mechanical configuration of the dual-mass system that maximizes the energy harvested of the incoming flow. To this end, a simplified two-degree-of-freedom mathematical model is introduced, where fluid forces are described taking into account the quasi-steady hypothesis, as suggested by Parkinson [29]. The mathematical model is approximately solved by applying the standard Harmonic Balance Method, and analyzed in detail. As a novelty, the analysis is mainly focused on the impact of the dual-mass parameters (those related to the mass of the secondary mass and the stiffness of the elastic connection between the galloping body and the secondary mass) on the efficiency of energy harvested. Results indicate that there are dual-mass configurations which improve the energy harvested with respect to that of the single mass system as well as the existence of configurations in which the efficiency of the energy harvested maximized across in a broader amount of values of the incident flow velocity. In this sense, the system becomes more broadband which is an interesting result when involving real flows which may have varying incident velocities.

The paper is organized as follows: a mathematical model for the transverse galloping in a dual-mass system for the first configuration is presented in Section 2. The first-order Harmonic Balance Method (HBM1) is used to obtain analytical solutions of the mathematical model; the influence of mechanical parameters and fluid velocity on the energy harvested is also discussed in the same section. Section 3 deals with the same issues as the previous ones but considering the second and third configurations. Finally, conclusions and some design guidelines are provided in Section 4. 

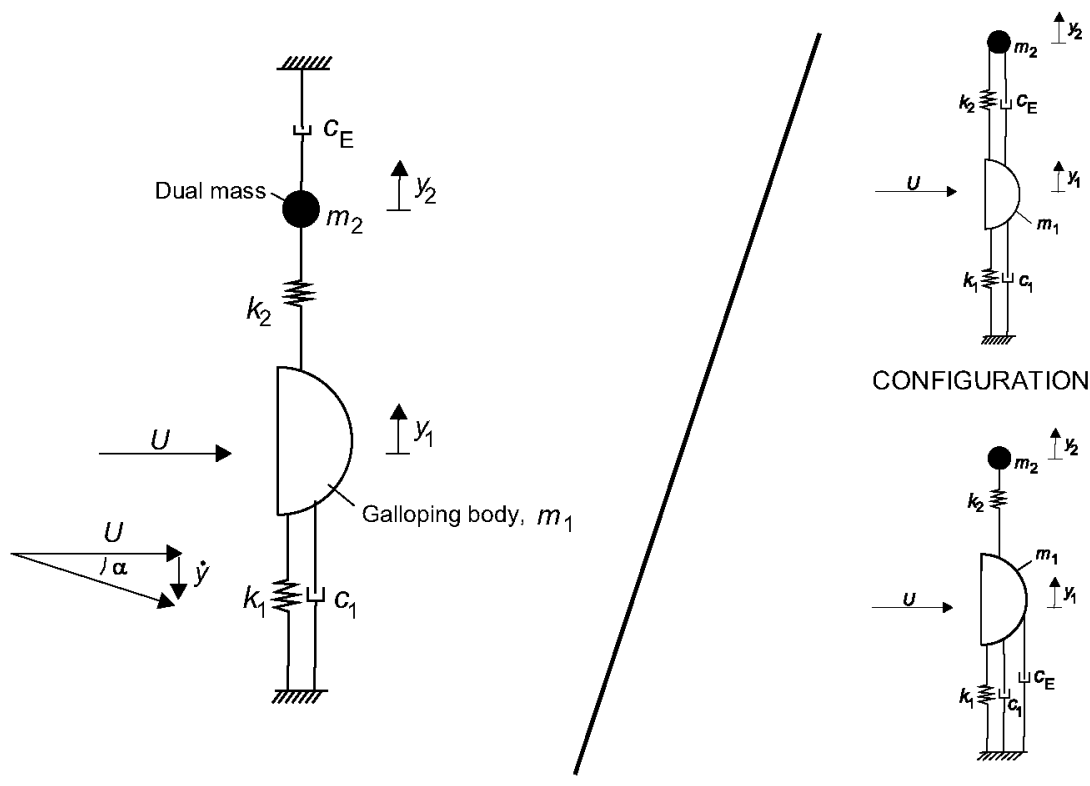

CONFIGURATION C2

CONFIGURATION C1

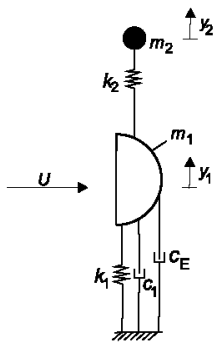

CONFIGURATION C3

Fig. 1. Defining sketch of the three possible dual-mass configurations which are differentiated by the situation of the harvesting damper.

\section{Mathematical model study for TG using a dual mass system for configuration C1}

A simplified dynamical system is considered, consisting of a spring-mounted prismatic body (galloping body) of mass $m_{1}$ immersed in an incoming flow and a secondary elastically supported mass $m_{2}$ (dual mass) which is not exposed to the aforementioned flow. They are linked by two linear springs, $k_{1}$ which connects the galloping body to a fix wall and $k_{2}$, which is situated between the galloping body and the dual mass (see Fig. $1 \mathrm{a}, \mathrm{C} 1$ configuration). The system is completed with a viscous damper, $c_{1}$, which is set between the fixed wall and the galloping body and an electrical generator which is represented, for simplicity, as another viscous damper $c_{E}$ located between the wall and the dual mass. This configuration can be understood as a two degree of freedom system where the prismatic body (galloping body) is subject to the influence of the incident flow and is prone to transverse galloping whereas the secondary mass (dual mass) does not suffer such influence and its vibrations are caused by its connection to the galloping body. All previous mechanical properties are defined per unit length of the galloping body.

\subsection{Mathematical model}

Balance among inertia, damping, stiffness, and fluid forces in the system yield the following system of two ordinary differential equations:

$$
\begin{gathered}
m_{1} \ddot{y}_{1}+c_{1} \dot{y}_{1}+k_{1} y_{1}+k_{2}\left(y_{1}-y_{2}\right)=\frac{1}{2} \rho U^{2} D C_{y}, \\
m_{2} \ddot{y}_{2}+k_{2}\left(y_{2}-y_{1}\right)+c_{E} \dot{y}_{2}=0,
\end{gathered}
$$

where $y_{1}$ denotes the position of galloping body, $y_{2}$ is the position of dual mass, with respect to the zero equilibrium position of the system, $\rho$ is the fluid density, $U$ is the undisturbed velocity of the incident flow, $D$ is the characteristic dimension of the cylinder prone to gallop normal to the flow, $C_{y}$ is the instantaneous fluid force coefficient on the galloping body in the transverse direction to the incident flow and finally, the dot symbol stands for differentiation with respect to physical time $t$.

In order to describe the fluid force coefficient on the galloping body, $C_{y}$, the quasi-steady hypothesis is resorted to, since it is assumed that the characteristic timescale of the galloping body oscillation is much larger than the characteristic timescale of the flow $\left(\left(k_{1} / m_{1}\right)^{-1 / 2} \geqslant 2 \pi D / U\right.$ ). According to this hypothesis, if there is no phase shift (or time lag) between the instantaneous fluid force coefficient $C_{y}$ (which is related to lift and drag as $C_{y}=-\left(C_{L}+C_{D} \tan \alpha\right) / \cos \alpha$ ) and the transverse body velocity, then the instantaneous fluid force acting on the moving body is nearly equal to the static force evaluated at the instantaneous angle of attack $\alpha$. Quasi-steady hypothesis works well when the reduced velocity is large enough, as discussed in detail in [29,32]. The functional relation between $C_{y}$ and $\alpha$ is typically expressed as a polynomial [20] and in this study, the order of the polynomial chosen is three. Polynomial fitting has been truncated to third order because it represents a reasonable compromise between development complexity and global accuracy of the predictions 
[30, p. 119]. The linear coefficient $a_{1}$ represents the slope of the vertical fluid force coefficient at zero angle of attack, and therefore it must be positive to trigger a galloping response. The coefficient $a_{3}$ accounts for the nonlinear effects and it has to be necessarily negative. From the previous considerations, the fluid force coefficient is

$$
C_{y}=\left(a_{1} \frac{\dot{y}_{1}}{U}+a_{3}\left(\frac{\dot{y_{1}}}{U}\right)^{3}\right),
$$

Introducing the following dimensionless variables, $\eta_{1}=y_{1} / D, \eta_{2}=y_{2} / D, \omega_{1}=\left(k_{1} / m_{1}\right)^{1 / 2}$, frequency ratio $\Omega=\left(k_{2} / k_{1}\right)^{1 / 2}$, dimensionless parasitic damping coefficient $\zeta_{1}=c_{1} /\left(2 m_{1} \omega_{1}\right)$ dimensionless damping coefficient of the generator $\zeta_{E}=c_{E} /\left(2 m_{1} \omega_{1}\right)$, mass ratio of the primary mass $m^{*}=m_{1} /\left(\rho D^{2}\right)$, ratio between masses $\mu=m_{2} / m_{1}$, dimensionless time $\tau=\omega_{1} t$, and reduced velocity $U^{*}=U /\left(\omega_{1} D\right)$ it is possible to write the dimensionless equations for the system dynamics:

$$
\begin{gathered}
\eta^{\prime \prime}{ }_{1}+2 \zeta_{1} \eta_{1}^{\prime}+\eta_{1}+\Omega^{2}\left(\eta_{1}-\eta_{2}\right)=\frac{U^{* 2}}{2 m^{*}}\left(a_{1} \frac{\eta_{1}^{\prime}}{U^{*}}+a_{3}\left(\frac{\eta_{1}^{\prime}}{U^{*}}\right)^{3}\right), \\
\mu \eta_{2}^{\prime \prime}+\Omega^{2}\left(\eta_{2}-\eta_{1}\right)+2 \zeta_{E} \eta_{2}^{\prime}=0
\end{gathered}
$$

where in this case the prime represents differentiation with respect to the dimensionless time. Note that the only external force exerted on the system is the fluid dynamic force.

\subsection{Galloping response}

Eqs. (3a) and (3b) contain six parameters $\left(\zeta_{1}, \zeta_{E}, \mu, \Omega, m^{*}\right.$ and $\left.U^{*}\right)$, meaning that the solution manifold exists inside a 6 -dimensional space. To gather a better understanding of the system behavior in this 6-dimensional space, the use of theoretical analysis able to yield analytical solutions (valid within the set of prescribed hypothesis) is deemed to be beneficial because it facilitates the visualization of the global trends. If it is assumed that the nonlinear term of the fluid force is small $\left(m^{*} U^{*} \geqslant 1\right)$ and that values of $\zeta_{E}$ are moderate, the Harmonic Balance Method of first order (HBM1) provides an approximate solution to Eqs. (3a) and (3b). The Harmonic Balance Method can be used when the solution of the problem is of a periodic nature. In practice, this means that an "ansatz" has to be made that needs to be verified after the said solution has been obtained. However, when compared to perturbation methods that do not require a priori restrictive hypothesis, this may seem kind of a drawback. On the other hand, tailoring the solution method to the specific nature of the solution itself has practical advantages regarding the numerical solution procedure; and this is the main reason why this approach has been chosen for this study. It is, nevertheless, to be mentioned that one of the authors, see Barrero-Gil et al. [23], has already used the "Krylov-Bogoliubov" perturbation method to address a problem on galloping phenomena. Assuming that the steady response is harmonical, solutions have the following form:

$$
\eta_{1}=A^{*} \sin \left(\omega^{*} \tau\right), \quad \eta_{2}=B^{*} \sin \left(\omega^{*} \tau+\phi\right),
$$

where the four unknowns, $\omega^{*}=\omega / \omega_{1}, A^{*}, B^{*}$ and $\phi$ have to be solved.

As previously stated, the resolution of the previous set of Eqs. (3a) and (3b) is straightforward. The novelty introduced in this paper comes from the study on the improvement of the efficiency of the energy harvested from the transverse galloping phenomenon when using a dual-mass system compared to the single mass case which is the main scope of the present article. From Eq. (3b), it is possible to obtain the relation between dual mass and galloping body amplitudes, as a linear two degrees of freedom response:

$$
K^{*}=\frac{B^{*}}{A^{*}}=\frac{\Omega^{2}}{\sqrt{\left(-\mu \omega^{* 2}+\Omega^{2}\right)^{2}+4 \zeta_{E}^{2} \omega^{* 2}}} .
$$

The phase delay $\phi$ of the harmonic response between the dual mass and the galloping body is given by

$$
\begin{aligned}
& \cos \phi=\frac{-\mu \omega^{* 2}+\Omega^{2}}{\sqrt{\left(-\mu \omega^{* 2}+\Omega^{2}\right)^{2}+4 \zeta_{E}^{2} \omega^{* 2}}}, \\
& \sin \phi=\frac{-2 \zeta_{E} \omega^{*}}{\sqrt{\left(-\mu \omega^{* 2}+\Omega^{2}\right)^{2}+4 \zeta_{E}^{2} \omega^{*^{2}}}} .
\end{aligned}
$$

All parameters involved for the determination of $K^{*}$ and $\phi$ depend only on mechanical properties $\left(\Omega, \mu, \zeta_{1}\right.$ and $\left.\zeta_{E}\right)$ which can be defined a priori at the design stage; the only unknown parameter is the dimensionless oscillating frequency $\omega^{*}$ which is obtained after applying HBM1 to Eq. (3a) and equating sine terms:

$$
D\left(\omega^{*}\right)=-\omega^{* 2}+1+\Omega^{2}\left(1-K^{*} \cos \phi\right)=0 .
$$

Eq. (7) can be solved numerically along with Eqs. (5) and (6), yielding $\omega^{*}$, which may have two solutions as it corresponds to a linear 2 degree of freedom system obtaining for each case different values of $K^{*}$ and $\phi$. In a steady-state solution, the system may only vibrate at one of the previous frequencies depending on the initial conditions of the system 
and in concordance, different galloping response as well as different values of energy harvesting efficiency may be obtained. Equating cosine terms in Eq. (3a) and truncating cubic cosine terms $\left[\cos \left(\omega^{*} \tau\right)^{3} \approx 3 / 4 \cos \left(\omega^{*} \tau\right)\right]$ the amplitude response $A^{*}$ is

$$
A^{*}=\left(\frac{4 U^{*}}{3 a_{3} \omega^{* 2}}\left(4 m^{*} \zeta_{T}-a_{1} U^{*}\right)\right)^{1 / 2}
$$

where $\zeta_{T}$ stands for

$$
\zeta_{T}=\zeta_{1}+\zeta_{E} K^{* 2}
$$

which is very useful in the ongoing discussion as it represents the equivalent damping of the whole dual mass system. $A^{*}$ depends on the reduced flow velocity $U^{*}$, the galloping body's geometry $\left(a_{1}\right.$ and $\left.a_{3}\right)$, the mechanical properties $\left(m^{*}, \mu\right.$, $\left.\Omega, \zeta_{1}\right)$, on the generator $\left(\zeta_{E}\right)$ and whether the system is vibrating at one or the other mode. In addition, note that $A^{*}$ has physical sense (i.e. $A^{*}>0$ ) when the reduced velocity exceeds a critical value $U_{g}^{* D \mathrm{DM}}$ which is the reduced velocity at which the system starts galloping meaning that the destabilizing effect of the fluid force equals the stabilizing effect of mechanical damping:

$$
U_{g}^{* \mathrm{DM}}=\frac{4 m^{*} \zeta_{T}}{a_{1}} .
$$

For fixed mechanical (or design) parameters, $\omega^{*}, K^{*}$ and $\phi$ are obtained numerically from Eqs. (5)-(7). Eq. (8) yields $A^{*}$, thus solving analytically the model of dual mass energy harvester. It is important to keep in mind that this solution is valid only whenever the quasi-steady hypothesis holds, when the effective reduced velocity $V^{*}=U^{*} / \omega^{*}$ is high enough. It has been checked that HBM1 approximation is quite robust, and comparisons with numerical solutions of Eq. (3) have been quite close even for values of $m^{*} U^{*}$ of order unity.

\subsection{Energy harvesting efficiency}

Once the solution of Eq. (3) has been derived, a conversion factor (or efficiency) is defined as the time-averaged power $P_{E}$ dissipated at $c_{E}$ divided by the flux of energy across the section of the prism:

$$
\eta^{\mathrm{DM}}=\frac{P_{E}}{\frac{1}{2} \rho U^{3} D},
$$

where $P_{E}$ is

$$
P_{E}=\frac{1}{T} \int_{0}^{T} c_{E} \dot{y}_{2}^{2} \mathrm{~d} t=2 m_{1} \omega_{1} \zeta_{E}\left(\omega_{1} D\right)^{2}\left\langle\dot{\eta}_{2}^{2}\right\rangle,
$$

and $T$ and $\langle\cdot\rangle$ mean respectively the time period of oscillation of the dual-mass system and the averaged value. It is possible to rewrite the efficiency $\eta^{\mathrm{DM}}$ as

$$
\eta^{\mathrm{DM}}=\frac{4 m^{*} \zeta_{E}\left\langle\dot{\eta}_{2}^{2}\right\rangle}{U^{* 3}}
$$

and from Eqs. (12) and (13) one gets $\eta^{\mathrm{DM}}$ by substituting the averaged value of $\left\langle\dot{\eta}_{2}^{2}\right\rangle$ :

$$
\eta^{\mathrm{DM}}=\frac{2 m^{*} \zeta_{E} B^{* 2} \omega^{* 2}}{U^{* 3}}
$$

Substituting $B^{*}$ (from Eqs. (5) and (8)) into Eq. (14) an analytical expression for the efficiency as a function of the mechanical properties $\left(\mu, \Omega, \zeta_{1}, \zeta_{E}\right)$, the geometry of the galloping body $\left(a_{1}, a_{3}\right)$ and the reduced velocity $U^{*}$ is obtained:

$$
\eta^{\mathrm{DM}}=\frac{8 m^{*} \zeta_{E}\left(4 m^{*} \zeta_{T}-a_{1} U^{*}\right) \Omega^{4}}{a_{3} U^{* 2}\left(\left(-\mu \omega^{* 2}+\Omega^{2}\right)^{2}+4 \zeta_{E}^{2} \omega^{* 2}\right)} .
$$

Differentiating Eq. (14) or Eq. (15) with respect to $U^{*}$ and equating to zero, the maximum efficiency is obtained at twice the critical velocity when the system starts galloping $\left(U_{\eta_{\max }^{* D M}}^{*}=2 U_{g}^{* \mathrm{DM}}\right)$.

$$
U_{\eta_{\max }^{* \mathrm{DM}}}^{*}=\frac{8 m^{*} \zeta_{T}}{a_{1}}
$$

Introducing $U_{\eta_{\max }}^{* \mathrm{DM}}$ into Eq. (15), the maximum efficiency can be obtained as

$$
\eta_{\max }^{\mathrm{DM}}=-\frac{a_{1}^{2}}{6 a_{3}} \frac{\zeta_{E}}{\zeta_{T}} K^{* 2}
$$




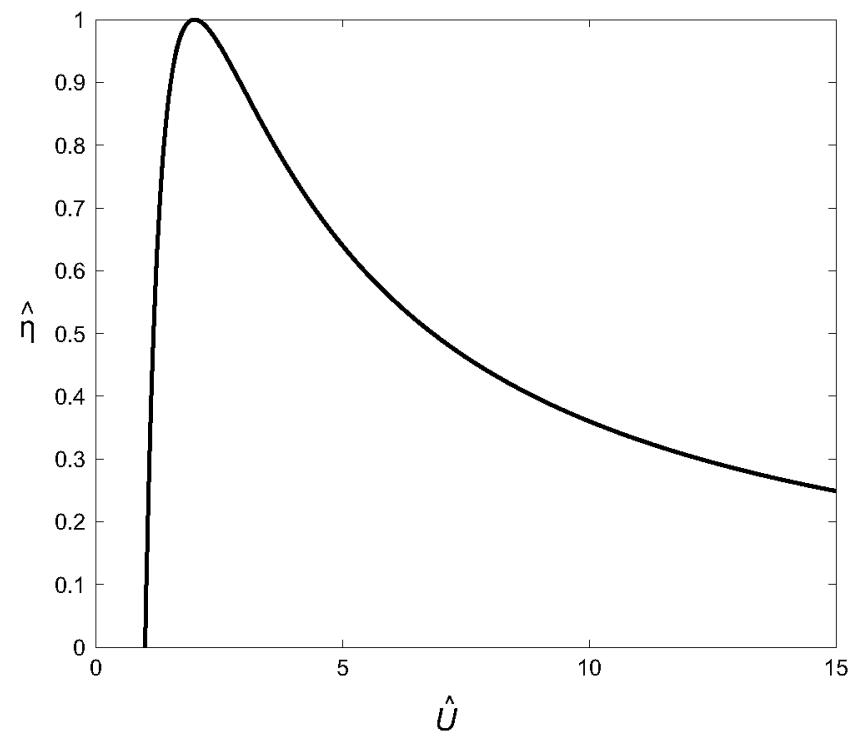

Fig. 2. Universal efficiency curve valid for all mechanical configurations. $\hat{\eta}=n / \eta_{\max }^{\mathrm{DM}}$ and $\hat{U}=U^{*} / U_{\mathrm{g}}^{* \mathrm{DM}}$.

A universal curve for the efficiency can be introduced using two new normalized variables, $\tilde{\eta}=\eta^{\mathrm{DM}} / \eta_{\max }^{\mathrm{DM}}$ and $\tilde{U}=U^{*} / U_{g}^{* \mathrm{DM}}$ and substituting them into Eq. (15)

$$
\tilde{\eta}=-\frac{4(1-\tilde{U})}{\tilde{U}^{2}}
$$

with these renormalized variables, it is possible to collapse the efficiency curves for all mechanical configurations into a single curve (Eq. (18)) providing information about the transverse galloping response over a range of reduced incident velocities. From Fig. 2, once galloping is started $(\tilde{U}=1)$, the normalized efficiency increases rapidly with the normalized reduced velocity until a value of $\tilde{U}=2$ is reached obtaining a value of the normalized efficiency of $\tilde{\eta}=1$, then the normalized efficiency diminishes slowly with $\tilde{U}$.

When the dual mass effect is minimized, for example making $\Omega=\infty, \mu=0$, the solution for the single mass configuration presented in Barrero-Gil et al. (2010) is recovered from (Eqs. (5)-(8) and Eq. (17):

$$
\begin{gathered}
K^{*}=\frac{B^{*}}{A^{*}}=1, \\
\phi=0, \\
\omega_{\mathrm{SM}}^{*}=1, \\
A_{\mathrm{SM}}^{*}=\left(\frac{4 U^{*}}{3 a_{3}}\left(4 m^{*}\left(\zeta_{1}+\zeta_{E}\right)-a_{1} U^{*}\right)\right)^{1 / 2}, \\
\eta_{\max }^{\mathrm{SM}}=-\frac{a_{1}^{2}}{6 a_{3}} \frac{\zeta_{E}}{\zeta_{1}+\zeta_{E}},
\end{gathered}
$$

In the ongoing discussion, the dual-mass system is compared to the single mass counterpart as defined by Eq. (19) in order to find mechanical configurations of $\mu$ and $\Omega$ that yield better energy harvesting efficiency.

\subsection{Dual mass effect}

From the practical side, so as to harness the maximum amount of energy, one would be interested in having (i) $\eta_{\max }^{\mathrm{DM}}$ as high as possible (maximum efficiency) and having (ii) $U_{g}^{* \mathrm{DM}}$ large in order to have a broadband character of efficiency dependence with the reduced velocity. Thus, two differentiated strategies exist for the design of the dual mass system. 


\subsubsection{Looking for high $\eta_{\max }^{\mathrm{DM}}$}

If there is no parasitic damping $\left(\zeta_{1}=0\right)$, from Eqs. (17) and (19e) one may see that the maximum available efficiency for the dual-mass and single-mass systems is

$$
\eta_{\max }^{\mathrm{DM}}=\eta_{\max }^{\mathrm{SM}}=-\frac{a_{1}^{2}}{6 a_{3}}=\eta_{H},
$$

where $\eta_{H}$ stands for the hydrodynamic efficiency which only depends on the geometry of the galloping body and therefore, when there is no parasitic damping, the maximum efficiency is $\eta_{H}$ for all possible mechanical configuration. In other words, it is not possible to extract power ideally $\left(\zeta_{1}=0\right)$, from the incoming flow, with better efficiency than for the single mass system using a dual-mass system. However, $\eta_{\max }^{\mathrm{DM}}$ and $\eta_{\max }^{\mathrm{SM}}$ cannot be (in general) as large as $\eta_{H}$ since the maximum power harnessable is less due to the dissipation of power as parasitic damping. This parasitic damping $\left(\zeta_{1}\right)$ can have relative importance (compared to $\zeta_{E}$ ) when building real transverse galloping energy harvesters. From Eqs. (17) and (19e) a harnessable factor that relates the hydrodynamic efficiency with the maximum harnessable efficiency for both single mass and dual-mass systems can be defined:

$$
\begin{gathered}
\gamma_{\mathrm{DM}}=\frac{\eta_{\max }^{\mathrm{DM}}}{\eta_{H}}=\frac{\zeta_{E} K^{* 2}}{\zeta_{T}}=\frac{\frac{\zeta_{E}}{\zeta_{1}} K^{* 2}}{1+\frac{\zeta_{E}}{\zeta_{1}} K^{* 2}}, \\
\gamma_{\mathrm{SM}}=\frac{\eta_{\max }^{\mathrm{DM}}}{\eta_{H}}=\frac{\frac{\zeta_{E}}{\zeta_{1}}}{1+\frac{\zeta_{E}}{\zeta_{1}}} .
\end{gathered}
$$

The goal of the ongoing discussion is to show that it is possible to enhance the dual-mass maximum efficiency compared to the single mass one when parasitic damping exists $\left(\zeta_{1} \neq 0\right)$. Thus, it is convenient to introduce a dual mass factor that relates the maximum efficiency of both dual and single mass systems:

$$
\psi_{\mathrm{DM}}=\frac{\eta_{\max }^{\mathrm{DM}}}{\eta_{\max }^{\mathrm{SM}}}=\frac{\gamma_{\mathrm{DM}}}{\gamma_{\mathrm{SM}}}=\frac{\left(1+\frac{\zeta_{E}}{\zeta_{1}}\right) K^{* 2}}{1+\frac{\zeta_{E}}{\zeta_{1}} K^{* 2}} .
$$

Observe that $\psi_{\mathrm{DM}}$ is not dependent on the geometry of the galloping body since this dependence is fully collected in $\eta_{H}$. If $\psi_{\mathrm{DM}}$ were larger than one then the corresponding dual-mass system would present a better maximum efficiency. From Eq. (23), the maximum value of $\psi_{\mathrm{DM}}^{\max }$ that can be obtained as

$$
\psi_{\mathrm{DM}}^{\max }=\gamma_{\mathrm{SM}}^{-1}=1+\frac{\zeta_{1}}{\zeta_{E}},
$$

which is the asymptotic value of Eq. (23) when $K^{*} \gg 1$. As it can be seen in Eq. (23), the improvement due to the dual-mass effect (that is $\psi_{\mathrm{DM}}>1$ ) comes from the fact that $K^{*}$ can be augmented beyond 1 . As both masses move at the same frequency, the power dissipated at each damper is proportional to $\zeta_{E} B^{* 2}$ and to $\zeta_{1} A^{* 2}$, and the system is capable of dissipating the same global power as in the single mass configuration, but proportionally the power dissipated in $\zeta_{E}$ has been enhanced as long as $K^{*}>1$. From Eq. (24), the potential improvement is larger as $\zeta_{1} / \zeta_{E}$ is increased, therefore the dual-mass configuration is indicated for situations in which $\zeta_{1} / \zeta_{E}$ is at least of the same order.

\subsubsection{Looking for a broadband-type response}

Real flows usually involve variations in the incident flow velocity. From a practical viewpoint it is interesting to have a broadband-type response with respect to variations of the incoming flow speed. As seen from the universal efficiency curve (see Fig. 2), high efficiency values are given around $\tilde{U}=2$. If it is desirable to have a normalized efficiency superior to a given one, for example, $\tilde{\eta}>x$ then the normalized range of reduced velocities where this happens is $\Delta \tilde{U}=\Delta U^{*} / U_{g}^{* D M}=4 x^{-1}(1-x)^{1 / 2}$ as obtained from Eq. (18). To analyze the broadband behavior of the dual-mass configuration, a new variable relating the critical galloping velocity of dual-mass and single-mass systems is introduced:

$$
\chi_{\mathrm{DM}}=\frac{U_{g}^{* \mathrm{DM}}}{U_{g}^{* \mathrm{SM}}}=\frac{1+\frac{\zeta_{E}}{\zeta_{1}} K^{* 2}}{1+\frac{\zeta_{E}}{\zeta_{1}}},
$$

which is only dependent on the mechanical parameters of the dual-mass system. Then the reduced galloping velocities that fulfill the prescribed condition for the efficiency is

$$
\Delta U^{*}=4 x^{-1}(1-x)^{1 / 2} U_{g}^{* \mathrm{DM}}=4 x^{-1}(1-x)^{1 / 2} U_{g}^{* S \mathrm{MM}} \chi_{\mathrm{DM}} .
$$


(a)

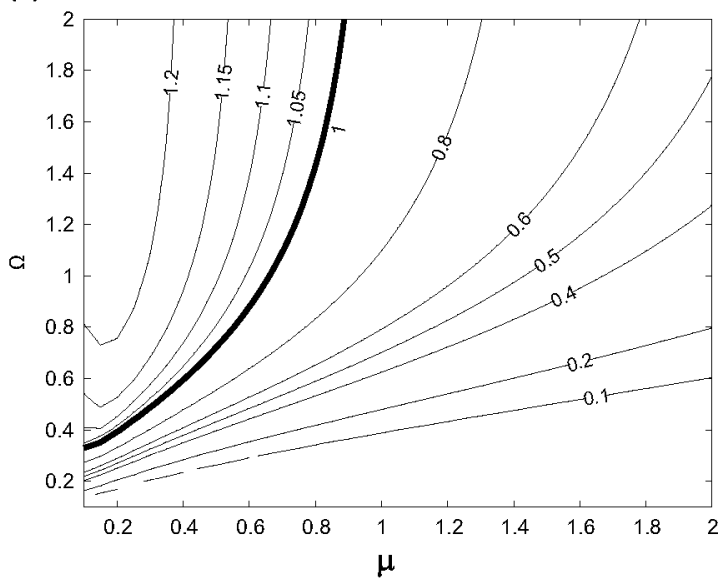

(b)

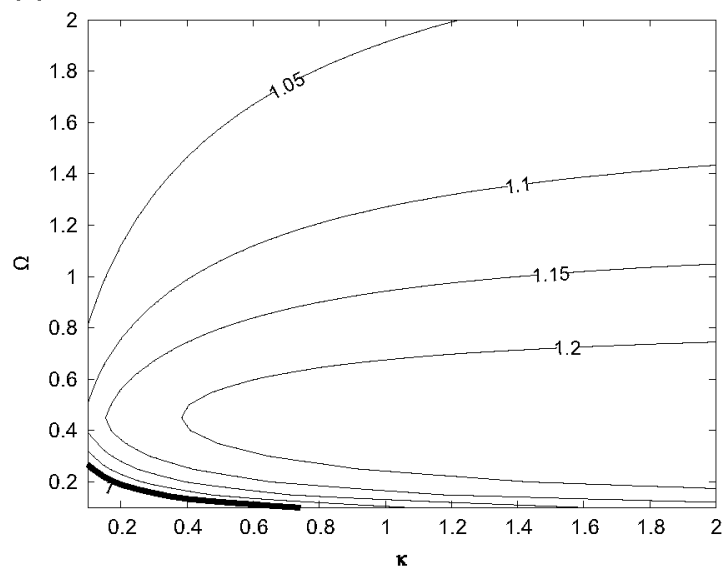

Fig. 3. $\psi_{\text {DM }}$ dependence with mechanical properties of the dual-mass systems $(\Omega, \mu)$ for the first (a) and the second (b) mode. The first mode always involves values of $\omega^{*}>1$ whereas the second one involves values of $\omega^{*}<1$

(a)

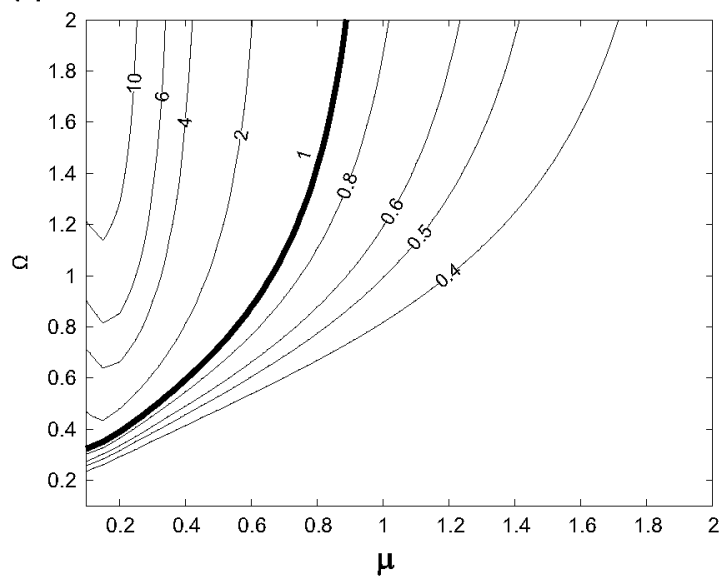

(b)

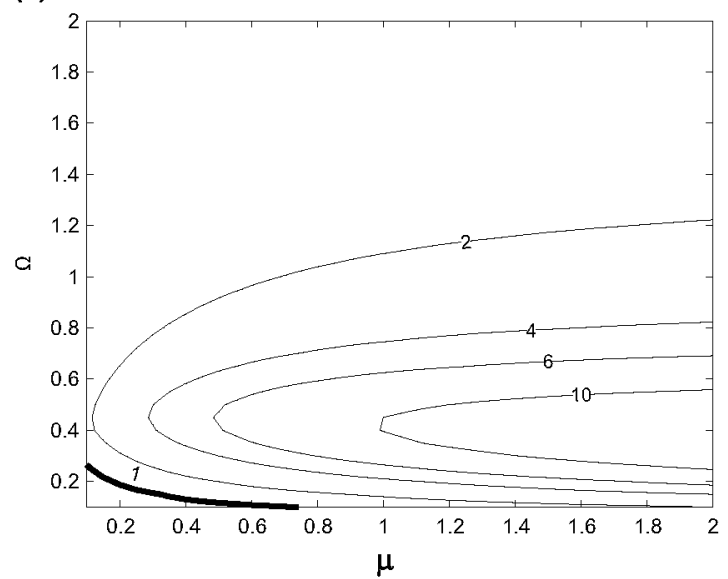

Fig. 4. $\chi_{\mathrm{DM}}$ dependence with mechanical properties of the dual-mass systems $(\Omega, \mu)$ for the first mode $\omega^{*}>1$ (a) and for the second mode $\omega^{*}<1$ (b).

This last equation shows that when $\chi_{\mathrm{DM}}>1$, the range of reduced velocities fulfilling the requested condition for the efficiency has been enlarged proportional to $\chi_{\mathrm{DM}}$ with respect to the single mass configuration and thus the dual-mass response is more broadband.

\section{5. $\psi_{\mathrm{DM}}$ and $\chi_{\mathrm{DM}}$ dependence on the mechanical parameters of the dual-mass system}

$\psi_{\mathrm{DM}}$ and $\chi_{\mathrm{DM}}$ give information about the properties of the dual-mass configuration in comparison to the original single mass system. They depend directly on the ratio of amplitudes between the dual mass and the galloping body $\left(K^{*}\right)$, on the parasitic damping $\left(\zeta_{1}\right)$ and on the generator $\left(\zeta_{E}\right)$. $K^{*}$, on the other hand, depends on $\mu, \Omega$ and $\zeta_{E}$ as well as the frequency $\left(\omega^{*}\right)$ at which the system is vibrating given by solving Eq. (7) from the two possible solutions (modes). Interesting configurations for $\mu$ and $\Omega$ are those that provide values of $\psi_{\mathrm{DM}}$ and $\chi_{\mathrm{DM}}$ larger than one, since it is desirable to enhance the maximum efficiency $\left(\psi_{\mathrm{DM}}>1\right)$ as well as to provide better efficiency for more values of the reduced velocity $\left(\chi_{\mathrm{DM}}>1\right)$.

Fig. 3 shows contour plots for $\psi_{\mathrm{DM}}$ as a function of $\Omega$ and $\mu$ for given values of $\zeta_{E}=0.05$ and $\zeta_{1}=0.0125$ and for each of the two possible modes. From Fig. 3a region can be observed in the parameter space $(\mu-\Omega)$ where $\psi_{\mathrm{DM}}$ is higher than one, meaning that the dual mass system tuned in that way increases the maximum efficiency achievable; note that values of $\psi_{\mathrm{DM}}$ under 1 means that the dual-mass systems introduce additional damping and behave like a traditional Tuned Mass Damper [25]. Values of $\zeta_{E}$ and $\zeta_{1}$ do not change the qualitative character of the results, but only the maximum achievable values.

With regard to $\chi_{\mathrm{DM}}$ behavior, from Fig. 4 it can be seen that there is also a region where $\chi_{\mathrm{DM}}$ is larger than one and thus the system is more broadband. On the other hand, $\chi_{\mathrm{DM}}<1$ means it is less broadband and therefore, for small changes in the reduced velocity from the optimal obtained at $U^{*}=2 U_{g}^{* \mathrm{DM}}$ lead rapidly to worse values of the efficiency. 
Table 1

Physical parameters for the single mass and dual-mass systems considered in the presented practical example.

\begin{tabular}{ll}
\hline Single mass & Dual mass \\
\hline$m^{*}=10$ & $m^{*}=10$ \\
$\zeta_{1}=0.0125$ & $\zeta_{1}=0.0125$ \\
$\zeta_{E}=0.05$ & $\zeta_{E}=0.05$ \\
- & $\mu=0.45$ \\
- & $\Omega=0.9$ \\
$a_{1}=0.79$ & $a_{1}=0.79$ \\
$a_{3}=-0.19$ & $a_{3}=-0.19$ \\
$D=0.05 \mathrm{~m}$ & $D=0.05 \mathrm{~m}$ \\
$L=1 \mathrm{~m}$ & $L=1 \mathrm{~m}$ \\
$\rho=1000 \mathrm{~kg} / \mathrm{m}^{3}$ & $\rho=1000 \mathrm{~kg} / \mathrm{m}^{3}$ \\
$\omega_{1}=2.5 \mathrm{rad} / \mathrm{s}$ & $\omega_{1}=2.5 \mathrm{rad} / \mathrm{s}$ \\
\hline
\end{tabular}

$\psi_{\mathrm{DM}}$ and $\chi_{\mathrm{DM}}$ behavior can be explained with the aid of Eqs. (23) and (25) since a causal relation between $\psi_{\mathrm{DM}}$ and $\chi_{\mathrm{DM}}$ with $K^{*}$ is derived

$$
\psi_{\mathrm{DM}} \chi_{\mathrm{DM}}=K^{* 2} \text {. }
$$

If $K^{*}>1$, then $\psi_{\mathrm{DM}}>1$ and $\chi_{\mathrm{DM}}>1$, whereas if $K^{*}<1, \psi_{\mathrm{DM}}$ and $\chi_{\mathrm{DM}}$ will also be smaller than one. Therefore, there are only two possible regions concerning $K^{*}$ as the trend of $\psi_{\mathrm{DM}}$ and $\chi_{\mathrm{DM}}$ only depend on this amplitude ratio.

The possibility of implementing more than two oscillating masses in the system has been analyzed using the same methodology (details are not described here for the sake of brevity). The conclusion is that neither the broadband character of the system response can be improved nor the peak efficiency increased with respect to the dual-mass oscillating system. This makes sense, since it is always possible to reduce the $N$-masses oscillating system to an equivalent dual-mass system.

\subsection{Practical example}

For illustrative purposes, a practical example is shown here. As it will be seen, thanks to the dual-mass arrangement, the maximum efficiency and the broadband character response with respect to a baseline single mass configuration are enhanced. Also, a comparison between the HBM1 method proposed and numerical solutions has been made. For this particular example, the single mass energy harvester used as a model has the following parameters: $m^{*}=10, \zeta_{1}=0.0125$ and $\zeta_{E}=0.05$ (for the whole definition of the system, see Table 1 ). This system could resemble a TG energy harvester immersed in water, as the mean density of the galloping body is slightly superior to the density of the water. The design criteria for the dual-mass $\mathrm{C} 1$ configuration have been that $\chi_{\mathrm{DM}} \approx 2$ and therefore, $U_{g}^{* \mathrm{DM}} \approx 2 U_{\mathrm{g}}^{* \mathrm{SM}}$. With the previous condition, values of $\mu=0.45$ and $\Omega=0.9$ are situated near the isoline for the value $\chi_{\mathrm{DM}}$ selected (see Fig. 4 ). With the chosen values of the mechanical parameters a value of $\psi_{\mathrm{DM}}=1.122$ is reached (see Fig. 3 ).

In Fig. 5a, the efficiency variation with the reduced velocity (flow speed) is plotted for the previous $\mathrm{C} 1$ configuration; this variation has been obtained with the HBM1 method as well as by numerical integration of Eqs. (3). Both solutions are close to each other and only a slight divergence between solutions can be observed for large values of $U^{*}$ which shows that the HBM1 method gives good approximations even when $m^{*} \sim 1$. Comparing the $\mathrm{C} 1$ dual-mass system to the original single mass one, the maximum efficiency has been improved by 12.2 percent. In addition, the efficiency dependence with the reduced velocity has been reduced in the sense that the system is more efficient in a larger range of reduced velocities; for example, in the single mass configuration $\eta$ is higher than 0.4 in the range $5<U^{*}<9$ approximately, whereas for the dualmass system the range of reduced velocities where $\eta>0.4$ is $8<U^{*}<21$ approximately. In Fig. 5b the dimensional power harvested is plotted as a function of the reduced velocity (flow speed). As it can observed, the power extracted is always superior (except for the initial velocities) for the dual-mass configuration. This example shows that it is possible to enhance the power extraction by changing the dual-mass parameters.

For illustration purposes and to gain better physical insight, a potential practical implementation of a dual-mass system is shown in Fig. 6. Note that the system displayed in the figure corresponds to the first configuration (C1). In operation the system would work as follows: under the action of the fluid flow, the galloping body oscillates perpendicular to the direction of the flow and drives the secondary mass (or dual mass). This secondary mass then drives the permanent magnet of a linear electromagnetic generator (note that for simplicity, the electromagnetic generator has been considered as a linear damper in the mathematical model, see [35]). Relative motion between the magnet and the coil of the generator produces electrical current. Note that a dual-mass system can be implemented in a previously built galloping energy harvester with small changes (which only includes the introduction of a secondary mass and reorganizing the elements that had already been installed in the original single mass system). Then, it is possible to improve the maximum efficiency that can be harvested as well as to broaden the range of reduced velocities where such efficiency is kept high. Thus, the dual-mass concept can theoretically improve the overall performance of the galloping energy harvester with minor modifications to the installation. 
(a)

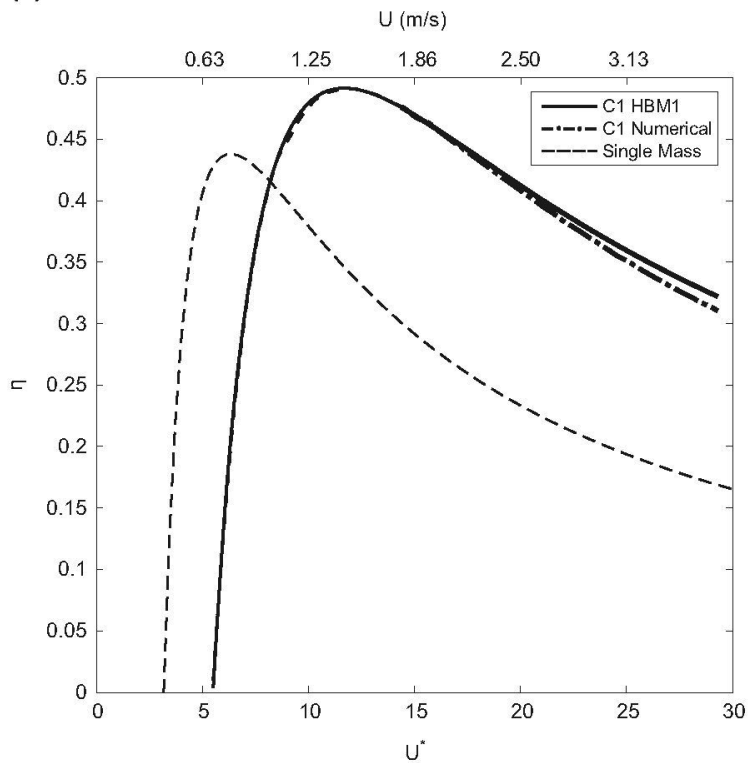

(b)

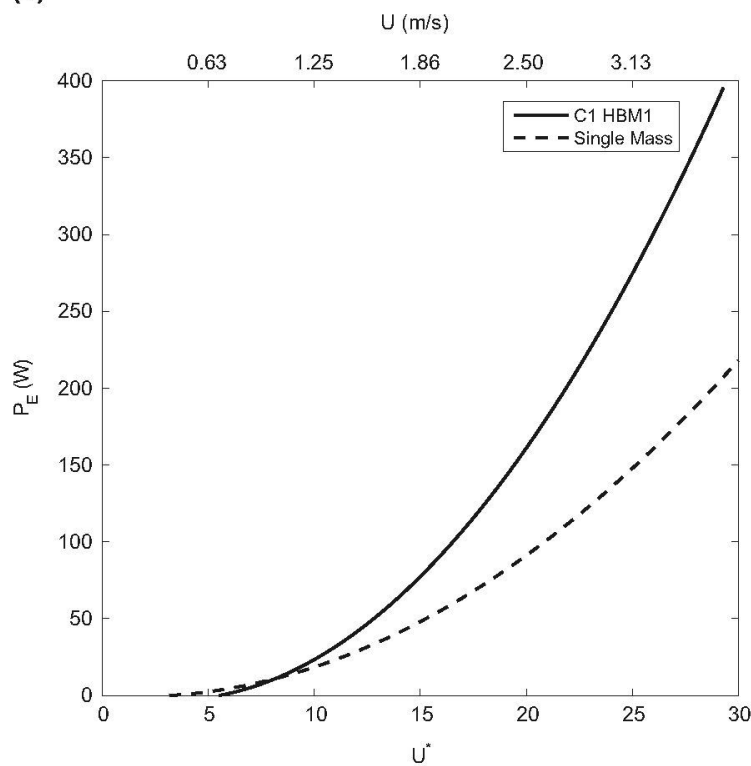

Fig. 5. Left: efficiency variation with the reduced velocity for the single mass system with the dual-mass system and dual-mass system (HBM1 and numerical solution). Right: power extracted for the single mass and dual-mass configurations.

(a)

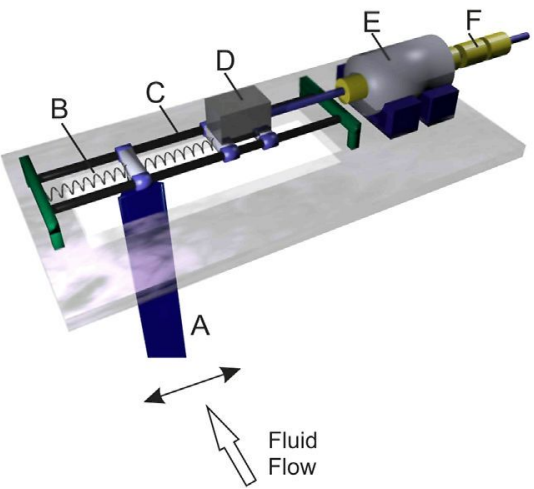

(b)

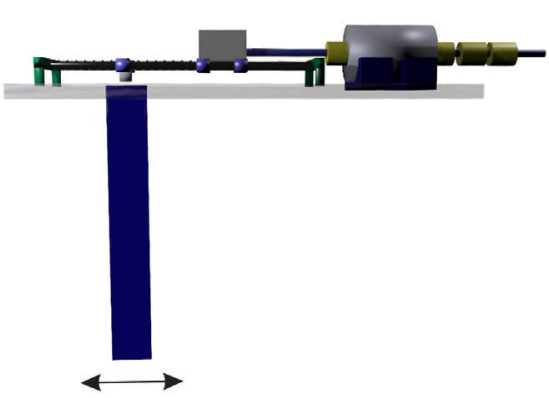

Fig. 6. Sketch showing a potential practical realization, which includes a galloping prism (A), springs (B), a linear guide (C) transverse to the flow direction, the secondary mass (D), the stator (coil) part of the electromagnetic generator, and a permanent magnet array (F). A 3D view is shown in (a) whereas a front view is shown in (b).

\section{Mathematical model study for TG using a dual mass system for configurations C2 and C3}

\subsection{C2 Configuration}

In this section the $\mathrm{C} 2$ configuration is presented as well as its most important results. For this case, the harvester damper $c_{E}$ is situated between the primary galloping body and the secondary mass (dual mass) (Fig. 1b). Having these changes present, the non-dimensional equations for the $\mathrm{C} 2$ configuration stands as such:

$$
\begin{gathered}
\eta_{1}^{\prime \prime}+2 \zeta_{1} \eta_{1}^{\prime}+\eta_{1}+2 \zeta_{E}\left(\eta_{1}^{\prime}-\eta_{2}^{\prime}\right)+\Omega^{2}\left(\eta_{1}-\eta_{2}\right)=\frac{U^{* 2}}{2 m^{*}}\left(a_{1} \frac{\eta_{1}^{\prime}}{U^{*}}+a_{3}\left(\frac{\eta_{1}^{\prime}}{U^{*}}\right)^{3}\right), \\
\mu \eta^{\prime \prime}{ }_{2}+2 \zeta_{E}\left(\eta_{2}^{\prime}-\eta_{1}^{\prime}\right)+\Omega^{2}\left(\eta_{2}-\eta_{1}\right)=0
\end{gathered}
$$


Applying the HBM1 method supposing harmonic solutions such as those of Eq. (4) the following solutions are obtained for Eq. (28). Solving in first place Eq. (38),

$$
\begin{gathered}
K^{*}=\frac{B^{*}}{A^{*}}=\frac{\sqrt{\Omega^{4}+4 \zeta_{E}^{2} \omega^{* 2}}}{\sqrt{\left(-\mu \omega^{* 2}+\Omega^{2}\right)^{2}+4 \zeta_{E}^{2} \omega^{* 2}}}, \\
\sin \phi=-\frac{2 \zeta_{E} \mu \omega^{* 3}}{\left(\Omega^{4}+4 \zeta_{E}^{2} \omega^{* 2}\right)^{1 / 2}\left(\left(\Omega^{2}-\mu \omega^{* 2}\right)^{2}+4 \zeta_{E} \omega^{* 2}\right)^{1 / 2}}, \\
\cos \phi=-\frac{\Omega^{2}\left(\Omega^{2}-\mu \omega^{* 2}\right)-4 \omega^{* 2} \zeta_{E}^{2}}{\left(\Omega^{4}+4 \zeta_{E}^{2} \omega^{* 2}\right)^{1 / 2}\left(\left(\Omega^{2}-\mu \omega^{* 2}\right)^{2}+4 \zeta_{E} \omega^{* 2}\right)^{1 / 2}},
\end{gathered}
$$

$K^{*}$ and $\phi$ are known for given $\zeta_{1}, \zeta_{E}, \mu$ and $\Omega$, except for $\omega^{*}$. Applying HBM1 to Eq. (36) and equating sine terms,

$$
D\left(\omega^{*}\right)=-\omega^{* 2}+1+\Omega^{2}\left(1-K^{*} \cos \phi\right)+2 \zeta_{E} K^{*} \sin \phi=0,
$$

Eq. (30) is an implicit function for $\omega^{*}$, which has two solutions corresponding to each of the possible modes. Equating cosine terms of (36) and truncating cubic terms as previously done,

$$
A^{*}=\left(\frac{4 U^{*}}{3 a_{3} \omega^{* 2}}\left(4 m^{*} \zeta_{T}-a_{1} U^{*}\right)\right)^{1 / 2} .
$$

The system starts to oscillate when the reduced velocity surpasses the critical galloping reduced velocity:

$$
U_{g}^{* \mathrm{DM}}=\frac{4 m^{*} \zeta_{T}}{a_{1}}
$$

expressions of Eqs. (31) and (32) are the same as for the $\mathrm{C} 1$ configuration. Only the value of $\zeta_{T}$ changes from the $\mathrm{C} 1$ to the $\mathrm{C} 2$ configuration, for this case:

$$
\zeta_{T}=\zeta_{1}+\zeta_{E}\left(1+K^{* 2}-2 K^{*} \cos \phi\right)
$$

\subsection{Energy harvesting efficiency}

The mean power $P_{E}$ dissipated at $c_{E}$ (now situated between the galloping body and the dual mass) is

$$
P_{E}=\frac{1}{T} \int_{0}^{T} c_{E}\left(\dot{y}_{1}-\dot{y}_{2}\right)^{2} \mathrm{~d} t=2 m_{1} \omega_{1} \zeta_{E}\left(\omega_{1} D\right)^{2}\left\langle\left(\dot{\eta}_{1}-\dot{\eta}_{2}\right)^{2}\right\rangle,
$$

where $\langle\cdot\rangle$ means the averaged value, the efficiency is then given as

$$
\eta^{\mathrm{DM}}=\frac{2 m^{*} \zeta_{E} A^{* 2}\left(1+K^{* 2}-2 K^{*} \cos \phi\right)}{U^{* 3}},
$$

The maximum efficiency occurs again at twice the critical velocity when the system starts galloping $\left(U_{\eta_{\max }}^{* \mathrm{DM}}=2 U_{g}^{* \mathrm{DM}}\right)$, Introducing the definition of critical velocity into Eq. (35), the maximum efficiency can be obtained as

$$
\eta_{\max }^{\mathrm{DM}}=-\frac{a_{1}^{2}}{6 a_{3}} \frac{\zeta_{E}}{\zeta_{T}}\left(1+K^{* 2}-2 K^{*} \cos \phi\right) .
$$

The discussion of the maximum harnessable energy is the same as for the $\mathrm{C} 1$ configuration. If there is no parasitic damping $\left(\zeta_{1}=0\right)$, then the maximum efficiency is

$$
\eta_{\max }^{\mathrm{DM}}=\eta_{\max }^{\mathrm{SM}}=-\frac{a_{1}^{2}}{6 a_{3}}=\eta_{H}
$$

whereas if $\zeta_{1} \neq 0$, then the maximum harnessable power will be less due to the dissipation of power in the parasitic damping. Dual-mass factors $\left(\psi_{\mathrm{DM}}, \chi_{\mathrm{DM}}\right)$ relating the maximum efficiency and the critical galloping velocity of the $\mathrm{C} 2$ configuration to the single mass harvester are also given:

$$
\psi_{\mathrm{DM}}=\frac{\gamma_{\mathrm{DM}}}{\gamma_{\mathrm{SM}}}=\frac{\left(\zeta_{1}+\zeta_{E}\right)\left(1+K^{* 2}-2 K^{*} \cos \phi\right)}{\zeta_{1}+\zeta_{E}\left(1+K^{* 2}-2 K^{*} \cos \phi\right)},
$$


(a)

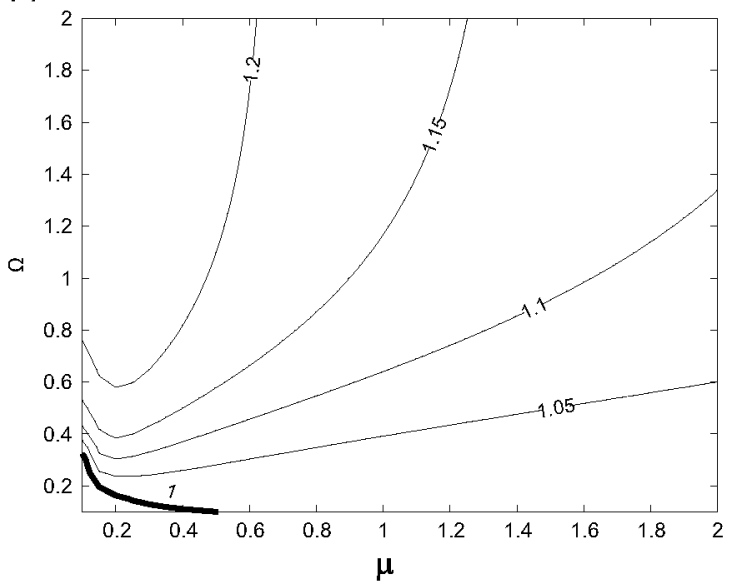

(b)

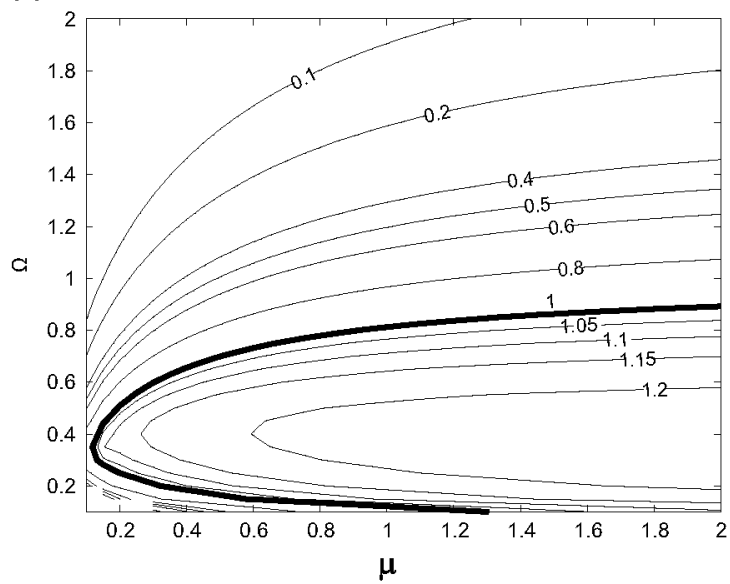

Fig. 7. (a) Variation of $\psi_{\mathrm{DM}}$ with $\Omega$ and $\mu$ for the $\mathrm{C} 2$ configuration ( $\omega^{*}>1$ mode). (b) Variation of $\psi_{\mathrm{DM}}$ with $\Omega$ and $\mu$ for the $\mathrm{C} 2$ configuration ( $\omega^{*}<1$ mode).

(a)

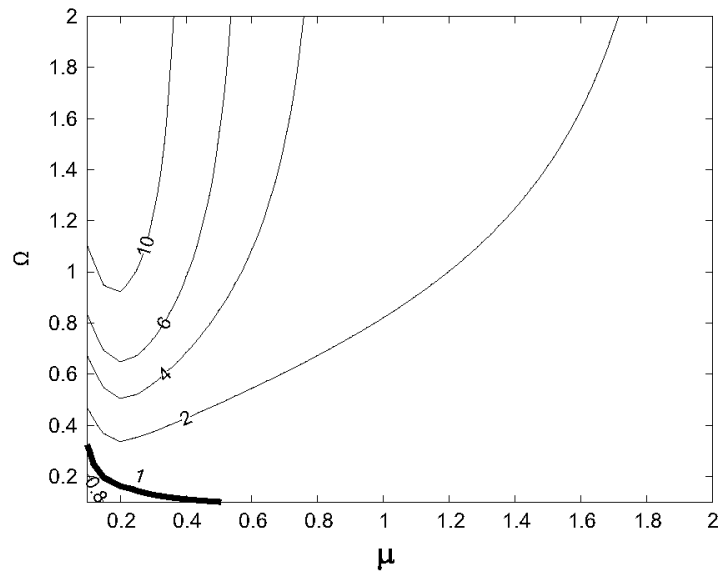

(b)

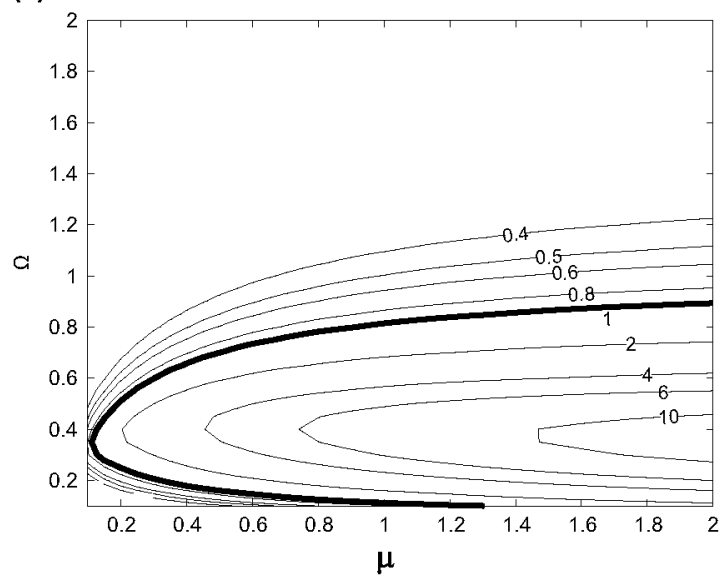

Fig. 8. Variation of $\chi_{\mathrm{DM}}$ with $\Omega$ and $\mu$ for the $\mathrm{C} 2$ configuration ( $\omega^{*}>1$ mode). (b) Variation of $\chi_{\mathrm{DM}}$ with $\Omega$ and $\mu$ for the $\mathrm{C} 2$ configuration ( $\omega^{*}<1$ mode).

$$
\chi_{\mathrm{DM}}=\frac{U_{g}^{* \mathrm{DM}}}{U_{g}^{* \mathrm{SM}}}=\frac{\zeta_{1}+\zeta_{E}\left(1+K^{* 2}-2 K^{*} \cos \phi\right)}{\left(\zeta_{1}+\zeta_{E}\right)},
$$

and the relationship between $\chi_{\mathrm{DM}}$ and $\psi_{\mathrm{DM}}$ is

$$
\psi_{\mathrm{DM}} \chi_{\mathrm{DM}}=1+K^{* 2}-2 K^{*} \cos \phi
$$

Eqs. (38) and (39) are of the same type as Eqs. (23) and (25), the only difference is that for the C1 configuration, the trend of $\psi_{\mathrm{DM}}$ and $\chi_{\mathrm{DM}}$ was given by $K^{*}$, whereas for the $\mathrm{C} 2$ configuration this trend is given now by $1+K^{* 2}-2 K^{* 2} \cos \phi$. If this parameter is bigger than unity, then both $\psi_{\mathrm{DM}}$ and $\chi_{\mathrm{DM}}$ are larger than unity, otherwise, they will be less than unity. Therefore, the $\mathrm{C} 2$ configuration yields the same qualitative behavior as for the $\mathrm{C} 1$ configuration. In other words, if $\psi_{\mathrm{DM}}^{\mathrm{C} 1}=\psi_{\mathrm{DM}}^{\mathrm{C} 2}$ then $\chi_{\mathrm{DM}}^{\mathrm{C} 1}=\chi_{\mathrm{DM}}^{\mathrm{C}}$. The main difference is that the previous values are obtained at different parameters of $\mu-\Omega$ at each configuration. The improvement in the $\mathrm{C} 2$ configuration's energy harvesting (as for the $\mathrm{C} 1$ ) comes from the fact that the power dissipated in $\zeta_{E}$ can be enhanced in detriment of $\zeta_{1}$ for correct values of $\mu$ and $\Omega$. In Figs. 7 and 8, contour plots are given as a function of $\mu$ and $\omega$ for values of $\zeta_{1}=0.0125$ and $\zeta_{E}=0.05$ for $\psi_{\mathrm{DM}}$ and $\chi_{\mathrm{DM}}$.

\subsection{C3 configuration}

The third configuration consists of the same elements as for the previous configurations, except that the generator represented by the damper $c_{E}$ is situated between a fixed wall and the galloping body in parallel to $c_{1}$ (see Fig. 1 ). This configuration is not of practical interest as it only yields similar results to the single mass configuration. As studied for the $\mathrm{C} 1$ and $\mathrm{C} 2$ configurations, the maximum efficiency cannot be enhanced beyond $\eta_{\max }^{\mathrm{DM}}=\eta_{H}$, only a distribution of where the 
energy is dissipated between the parasitic damping or the generator can be obtained, and as $c_{1}$ and $c_{E}$ are situated in parallel, then for all mechanical configurations

$$
\begin{gathered}
\gamma_{\mathrm{DM}}=\gamma_{\mathrm{SM}}=\frac{\zeta_{E}}{\zeta_{1}+\zeta_{E}} \Rightarrow \psi_{\mathrm{DM}}=1, \\
U_{\mathrm{g}}^{* \mathrm{DM}}=U_{\mathrm{g}}^{* \mathrm{SM}} \Rightarrow \chi_{\mathrm{DM}}=1 .
\end{gathered}
$$

\section{Concluding remarks}

This paper considers a dual-mass energy harvester in order to take advantage of transverse galloping oscillations from a fluid flow. An analytical model has been derived in order to comprehend its potential advantages. The solution exists in a $6-$ dimensional space which includes parameters such as the reduced flow velocity $U^{*}$, mass ratio $m^{*}$, parasitic damping $\zeta_{1}$, the harvesting generator $\zeta_{E}$ and two dual mass mechanical parameters, the frequency ratio $\Omega$ and the masses ratio $\mu$. Such a model sheds physical insight to the problem in order to comprehend its potential advantages compared to a single mass case. Some important results have been obtained, the maximum efficiency for each configuration always happens at twice the initial galloping velocity and the maximum efficiency is equivalent to the original single mass system multiplied by a factor that depends solely on $\mu, \Omega$ and $\zeta_{E}$ and a universal curve valid for all dual-mass configurations as well as for the single mass systems has been presented. Three possible configurations have been analyzed obtaining that the maximum efficiency cannot surpass that of the single mass system when there is no parasitic damping, but when there is parasitic damping, the efficiency can be enhanced for $\mathrm{C} 1$ and $\mathrm{C} 2$ configurations importantly if the value of the parasitic damping is similar to the harvesting damper. To this end, mechanical parameters of the dual-mass system, namely the frequency ratio $\Omega$ and the masses ratio $\mu$, have to be chosen appropriately.

An important issue when dealing with real flows is the variation of the flow velocity and how it affects the efficiency achievable. In this sense, for the $\mathrm{C} 1$ and $\mathrm{C} 2$ configurations, it has been obtained that it is possible to broaden the values of the incident velocities at which the efficiency has been kept higher, making the efficiency achievable less dependent on flow variations. It must be noted that the analysis carried out is an approximation to the real problem, nonetheless this paper's results are of interest in order to design real devices which have parasitic losses and to extract energy from transverse galloping in an efficient way for real varying flows.

\section{Acknowledgments}

This research has been funded by the international company REPSOL SA, through the REPSOL-INSPIRE program and under Contract P120130288.

\section{References}

[1] M.M. Bernitsas, K. Raghawan, VIVACE (Vortex Induced Vibration for Aquatic Clean Energy): a new concept in generation of clean and renewable energy from fluid flow, Journal of Offshore Mechanics and Arctic Engineering, ASME Transactions 34 (2008) 34.

[2] K. Raghavan, M.M. Bernitsas, D. Maroulis, Effect of Reynolds number on vortex induced vibrations, IUTAM Symposium, Hamburg, Germany, July 2007.

[3] C. Grouthier, S. Michelin, E. De Langre, Energy Harvesting by Vortex-Induced Vibrations in Slender Structures, Paper No. OMAE2013-10241, 2013, http://dx.doi.org/10.1115/OMAE2013-10241.

[4] A.W. Mackowski, C.H.K. Williamson, An experimental investigation of vortex-induced vibration with nonlinear restoring forces, Physics of Fluids 25 (8) (2013) 087101.

[5] A. Barrero-Gil, S. Pindado, S. Avila, Extracting energy from vortex-induced vibrations: a parametric study, Applied Mathematical Modelling 36 (7) (2012) 3153-3160.

[6] M. Sanchez-Sanz, B. Fernandez, A. Velazquez, Energy-harvesting microresonator based on the forces generated by the Karman street around a rectangular prism, Journal of Microelectromechanical Systems 18 (2009) 449-457.

[7] A. Abdelkefi, M.R. Hajj, A.H. Nayfeh, Phenomena and modeling of piezoelectric energy harvesting from freely oscillating cylinders, Nonlinear Dynamics 70 (2) (2012) 1377-1388.

[8] A. Mehmood, A. Abdelkefi, M.R. Hajj, A.H. Nayfeh, I. Akhtar, A.O. Nuhait, Piezoelectric energy harvesting from vortex-induced vibrations of circular cylinder, Journal of Sound and Vibration 332 (19) (2013) 4656-4667.

[9] H.L. Dai, A. Abdelkefi, L. Wang, Theoretical modeling and nonlinear analysis of piezoelectric energy harvesting from vortex-induced vibrations, Journal of Intelligent Material Systems and Structures (2014), http://dx.doi.org/10.1177/1045389X14538329.

[10] H.L. Dai, A. Abdelkefi, L. Wang, Piezoelectric energy harvesting from concurrent vortex-induced vibrations and base excitations, Nonlinear Dynamics 77 (2014) 967-981.

[11] H.D. Akaydin, N. Elvin, Y. Andreopoulos, The performance of a self-excited fluidic energy harvester, Smart Materials and Structures 21 (2012) 025007.

[12] Y. Nishi, Y. Ueno, M. Nishio, L.A. Rodrigues Quadrante, K. Kokubun, Power extraction using flow-induced vibration of a circular cylinder placed near another fixed cylinder, Journal of Sound and Vibration 333 (2014) 2863-2880.

[13] H.J. Jung, S.W. Lee, The experimental validation of a new energy harvesting system based on the wake galloping phenomenon, Smart Mater, Struct. 20 (2011) 055022.

[14] A. Abdelkefi, J.M. Scanlon, E. McDowell, M.R. Hajj, Performance enhancement of piezoelectric energy harvesters from wake galloping, Applied Physics Letters 103 (2013) 033903.

[15] L. Tang, P. Paidoussis, J. Jang, Cantilevered flexible plates in axial flow: energy transfer and the concept of flutter-mill, Journal of Sound and Vibration 326 (1-2) (2009) 263-276. 
[16] Q. Zhu, M. Haase, C.H. Wu, Modeling the capacity of a novel flow-energy harvester, Applied Mathematical Modelling 33 (2009) $2207-2217$.

[17] M. Bryant, E. Garcia, Modeling and testing of a novel aeroelastic flutter energy harvester, Journal of Vibration and Acoustics 133 (2011) 011010.

[18] A. Abdelkefi, A.H. Nayfeh, M.R. Hajj, Modeling and analysis of piezoaeroelastic energy harvesters, Nonlinear Dynamics 67 (2012) $925-939$.

[19] J.S. Bae, D.J. inman, Aeroelastic characteristics of linear and nonlinear piezo-aeroelastic energy harvester, Journal of Intelligent Material Systems and Structures 25 (4) (2014) 401-416.

[20] A. Abdelkefi, A.O. Nuhait, Modeling and performance analysis of cambered wing-based piezoaeroelastic energy harvesters, Smart Materials and Structures 22 (2012) 095029.

[21] J.J. Allen, A.J. Smits, Energy harvesting eel, Journal of Fluids and Structures 15 (2001) 629-640.

[22] G.W. Taylor, J.R. Burns, S.M. Kammann, W.B. Powers, T.R. Welsh, The energy harvesting Eel: a small subsurface ocean/river power generator, IEEE Journal of Oceanic Engineering 26 (4) (2001) 539-547.

[23] A. Barrero-Gil, A. Sanz-Andres, G. Alonso, Energy harvesting from transverse galloping, Journal of Sound and Vibration 329 (14) (2010) $2873-2883$.

[24] A. Abdelkefi, M.R. Hajj, A.H. Nayfeh, Power harvesting from transverse galloping of square cylinder, Nonlinear Dynamics 70 (2) (2012) $1355-1363$.

[25] A. Abdelkefi, M.R. Haj, A.H. Nayfeh, Piezoelectric energy harvesting from transverse galloping of bluff bodies, Smart Materials and Structures 22 (1) (2013) 015014

[26] A. Abdelkefi, Z. Yan, M.R. Haji, Temperature impact on the performance of galloping-based piezoaeroelastic energy harvesters, Smart Materials and Structures 22 (5) (2013) 055026.

[27] J. Sirohi, R. Mahadik, Harvesting wind energy using a galloping piezoelectric beam, Journal of Vibration and Acoustics 134 (1) (2011) 011009.

[28] L. Zhao, L. Tang, Y. Yang, Small wind energy harvesting from galloping using piezoelectric materials, ASME 2012 Conference on Smart Materials, Adaptive Structures and Intelligent Systems, http://dx.doi.org/10.1115/SMASIS2012-8212.

[29] G.V. Parkinson, Phenomena and modelling of flow-induced vibrations of bluff bodies, Progress in Aerospace Sciences 26 (2) (1989) $169-224$.

[30] R. Blevins, Flow-Induced Vibrations, Van Nostrand Reinhold, Amsterdam, 1990.

[31] E. Naudascher, D. Rockwell, Flow-Induced Vibrations an Engineering Guide, Dover Publications, New York, 1994.

[32] M.P. Paidoussis, J.P. Stuart, E. De Langre, Fhiid-Structure Interactions, Cambridge University Press, New York, 2011.

[33] X. Tang, L. Zuo, Enhanced vibration energy harvesting using dual-mass systems, Journal of Sound and Vibration 330 (2011) $5199-5209$.

[34] Y. Nishi, Power extraction from vortex-induced vibration of dual mass system, Journal of Sound and Vibration 332 (2013) 199-212.

[35] D. Vicente-Ludlam, A. Barrero-Gil, A. Velazquez, Optimal electromagnetic energy extraction from transverse galloping, Journal of Fluids and Structures (2014), http://dx.doi.org/10.1016/j.jfluidstructs.2014.09.007. 